\title{
L'ÉVALUATION PRÉALABLE DANS LES CONTRATS DE PARTENARIAT
}

\author{
Claude MÉNARD \\ Professeur à l'Université de Paris (Panthéon-Sorbonne)
}

Jean-Michel OUDOT

Chargé d'études économiques à la direction des affaires financières du ministère de la défense, enseignant à Sciences Po Paris et chercheur associé à l'Université de Paris (Panthéon-Sorbonne)

\section{REVUE FRANCAISE D'ADMINISTRATION PUBLIQUE}

NO 130, ETE 2009, PP. 349-364 


\title{
L'ÉVALUATION PRÉALABLE DANS LES CONTRATS DE PARTENARIAT
}

\author{
Claude MÉNARD
}

Professeur à l'Université de Paris (Panthéon-Sorbonne)

\begin{abstract}
Jean-Michel OUDOT
Chargé d'études économiques à la direction des affaires financières du ministère de la défense, enseignant à Sciences Po Paris et chercheur associé à l'Université de Paris (Panthéon-Sorbonne)
\end{abstract}

\begin{abstract}
Résumé
La comparaison de solutions contractuelles alternatives est obligatoire, en France, pour justifier le recours au contrat de partenariat. La question se pose alors des fondements d'une telle comparaison. La théorie des coûts de transaction fournit des outils particulièrement éclairants pour examiner la pertinence relative des procédures mises en œuvre à cet effet. Cet article s'appuie sur cette théorie et sa confrontation à des évaluations existantes pour explorer un certain nombre de pistes susceptibles de rapprocher les conditions de signature et d'exécution des marchés des termes résultant de l'évaluation préalable.
\end{abstract}

$1 \quad$ Cet article s'inspire d'un rapport remis au Centre d'expertise français pour l'observation des partenariats public-privé (CEF-O-PPP) en avril 2009. Sami Ben Ali, Julie de Brux, Laurent Garcin, Jérémie Gatignol et Marie-Joëlle Kodjovi sont remerciés pour l'aide apportée à la rédaction de ce rapport. Nous remercions aussi l'Institut de la Gestion Déléguée et la Mission d'Appui aux Partenariats Public-Privé (MAPPP) pour nous avoir facilité l'accès à leurs données, ainsi que deux rapporteurs anonymes de la RFAP pour leurs remarques constructives. Les co-auteurs de cet article restent bien entendu seuls responsables des analyses et opinions développées ici. 


\begin{abstract}
A comparative analysis of alternative contractual solutions is now required by the law, in France, to legitimize the choice of a Public-Private Partnership. This procedure is identified as "evaluation préalable" (preliminary evaluation). A central question then becomes that of the foundations of such comparisons. Transaction cost economics provides particularly powerful tools for scrutinizing the relevance of procedures implemented in that respect. This paper takes stock of this contribution and, through its confrontation to existing evaluations, it explores means for improving them and to facilitate a more coherent approach between this ex-ante analysis and the design and ex-post implementation of solutions chosen.
\end{abstract}

\title{
INTRODUCTION
}

La signature et la mise en œuvre des contrats de partenariat, tels que définis par l'ordonnance française de juin 2004, implique d'avoir préalablement justifié le recours à ce type d'arrangement. Une telle justification est formalisée dans l'évaluation préalable dont l'obligation a été confirmée par la loi n²008-735 du 28 juillet 2008 relative aux contrats de partenariat. En deçà de sa justification affichée, qui est essentiellement légale, cette étape précontractuelle entend aussi permettre une première reconstitution du coût complet du marché considéré de manière à prévoir les autorisations d'engagement correspondantes. Il y a donc là des enjeux majeurs. L'évaluation préalable s'avère pourtant délicate à mener. Elle impose en effet de comparer des solutions organisationnelles alternatives avant même que celles-ci ne soient mises en œuvre.

L'objectif de cet article est de proposer et de justifier des éléments méthodologiques susceptibles de faciliter la réalisation des évaluations préalables et d'en accroître la crédibilité. Nous nous sommes appuyés pour ce faire sur la littérature économique, juridique et de gestion, sur une étude attentive des documents disponibles concernant le principe et la pratique de l'évaluation préalable, et enfin sur une série d'entretiens ainsi que sur l'analyse 
détaillée de quelques évaluations préalables déjà réalisées en France ${ }^{2}$. Du point de vue théorique, l'analyse en termes de coûts de transaction s'est révélée particulièrement éclairante. Nous en dégageons plusieurs pistes visant à rapprocher les conditions de signature et d'exécution des marchés des termes retenus par les évaluations préalables, soit en améliorant leur qualité intrinsèque, soit en retenant les conclusions de l'analyse comme guide de négociation et d'exécution des marchés.

Nous développons ces idées en trois sections. La première rappelle certaines caractéristiques des évaluations préalables et les trois critères susceptibles d'être mobilisés pour apprécier la pertinence relative des solutions contractuelles alternatives. Une attention particulière est portée au critère d'efficacité économique, introduit en 2008, et qui ouvre des perspectives nouvelles au décideur public. La deuxième section fait le point sur certains aspects clés d'une telle analyse comparée, en faisant momentanément abstraction des risques. La troisième section est consacrée à la prise en compte des risques dans les évaluations préalables.

\section{CARACTERISTIQUES DE L'EVALUATION PREALABLE}

\section{Objectifs et acteurs}

L'évaluation préalable poursuit avant tout des objectifs d'ordre contractuel mais ouvre aussi la porte, par le biais du troisième critère en particulier, à des considérations budgétaires dont on peut penser qu'elles prendront une place croissante. Du point de vue contractuel, elle vise fondamentalement à identifier et justifier la solution organisationnelle la plus adaptée au

2 Projet d'éclairage public de la ville de Saint Fons ; projet de mise en place d'un système sophistiqué de régulation de circulation pour la ville de Rouen; projet d'établissement d'un réseau de communication électronique ouvert au public au profit du conseil régional d'Auvergne ; projet de réalisation d'un $3^{\mathrm{e}}$ lot de prisons (ministère de la justice). 
besoin exprimé par la personne publique, par exemple le choix entre marché public classique, délégation de services publics et contrat de partenariat. Il est attendu de cette comparaison qu'elle soit menée de façon objective, sans privilégier un arrangement plutôt qu'un autre. Le second objectif de l'évaluation préalable, celui-là non contraignant, est de présenter à la Direction du budget, pour les projets d'État, des éléments probants justifiant le coût global du marché considéré de manière à prévoir les autorisations d'engagements correspondantes. L'approche doit donc, en principe, assurer la prise en compte de l'ensemble des coûts.

Satisfaire ces deux objectifs devrait entraîner un certain nombre d'avantages, en particulier la définition précise du besoin exprimé, la réduction de l'asymétrie d'information entre les parties, et l'obtention d'un pré-programme fonctionnel, le tout servant de base à l'élaboration du programme à partir duquel les candidats élaboreront leurs offres. La passation et l'exécution du marché sont donc susceptibles d'être facilitées par l'évaluation préalable, ce qui accroît d'autant son utilité, quelle que soient les formes envisagées et la solution retenue.

La loi de 2008 définit le contrat de partenariat comme un « contrat administratif par lequel l'État ou un établissement public de l'État confie à un tiers, pour une période déterminée en fonction de la durée d'amortissement des investissements ou des modalités de financement retenues, une mission globale ayant pour objet le financement, la construction ou la transformation, l'entretien, la maintenance, l'exploitation ou la gestion d'ouvrages, d'équipements ou de biens immatériels nécessaires au service public » (art. 1).

De son côté, l'évaluation préalable a pour objet de faire « apparaître les motifs de caractère économique, financier, juridique et administratif qui conduisent la personne publique à engager la procédure de passation d'un tel contrat. [...] Cette évaluation comporte une analyse comparative de différentes options, notamment en termes de coût global hors taxes, de 
partage des risques et de performance, ainsi qu'au regard des préoccupations de développement durable » (art. 2, I).

L'évaluation préalable ainsi définie élargit le spectre des compétences susceptibles d'être mobilisées. Outre les administrations centrales ou les collectivités territoriales concernées, la Mission d'appui à la réalisation des partenariats public-privé (MAPPP) ainsi que des cabinets de conseil juridique et financier interviennent de façon beaucoup plus explicite que par le passé dans la procédure. Rappelons que la MAPPP, organisme du ministère de l'économie, de l'industrie et de l'emploi, a pour mission d'apporter à la personne publique un appui méthodologique, sans s'impliquer directement dans la mise en œuvre de l'évaluation préalable. Le recours aux cabinets de conseil a pour objectif de permettre à la personne publique de bénéficier de leur expertise. La diversité des compétences ainsi mobilisées, qui varie évidemment selon les projets envisagés, entend accroître l'efficacité de l'évaluation préalable.

\section{Trois critères de sélection}

Pour apprécier la pertinence respective des solutions à prendre en compte, l'évaluation préalable peut prendre appui sur trois critères. Soulignons qu'en vertu de l'article 2 de la loi de juillet 2008 et conformément à la décision du Conseil constitutionnel n²008-567, du 24 juillet 2008, ces critères peuvent être mobilisés de façon alternative.

\section{Le motiffondé sur la complexité}

Ce motif, instauré par l'ordonnance de 2004, est maintenu dans la loi de juillet 2008. Il tend à prévaloir dans la justification du recours aux contrats de partenariat, sans doute parce qu'il est plus facile à argumenter ou à défendre en cas de contestation. Il y a complexité lorsque la personne publique n'est pas objectivement en mesure de définir seule et à l'avance 
les moyens techniques pouvant répondre à ses besoins, ou d'établir le montage juridique ou financier du projet. La complexité du projet concerne les données intrinsèques de l'opération, c'est-à-dire les caractéristiques objectives de l'ouvrage ou du service. L'impossibilité de définir les moyens aptes à satisfaire les besoins ou d'évaluer ce que le marché peut offrir en termes de solutions techniques et / ou financières et / ou juridiques doit être objective, et non être due à une carence de la personne publique.

\section{Le motiffondé sur l'urgence}

Un deuxième critère, présent lui aussi dans l'ordonnance de 2004, a trait à l'urgence. Dans la loi du 28 juillet 2008, l'article 2 (paragraphe II alinéa 2) définit l'urgence en ces termes: « le projet présente un caractère d'urgence, lorsqu'il s'agit de rattraper un retard préjudiciable à l'intérêt général affectant la réalisation d'équipements collectifs ou l'exercice d'une mission de service public, quelles que soient les causes de ce retard, ou de faire face à une situation imprévisible ». Ce motif a été traduit de la façon suivante dans le guide sur les contrats de partenariat édité par le ministère de l'économie, des finances et de l'industrie : «Il y a urgence quand le besoin en construction ou rénovation d'équipements ou d'ouvrages résulte d'une cause extérieure à l'administration ». Une réponse ministérielle a par ailleurs précisé que l'urgence «en la matière n'est pas différente de celle traditionnellement admise par les juridictions administratives $»^{3}$. Ainsi, une personne publique ne peut invoquer, au titre de l'urgence, une situation résultant de carences manifestes de sa part, ou le seul souci «d'aller vite ». La jurisprudence du Conseil constitutionnel et du Conseil d'État conduit à penser que l'urgence peut résulter du constat d'un retard grave et préjudiciable, par exemple en matière d'éclairage public, auquel la personne publique est incapable de remédier dans des délais raisonnables par les voies classiques de la commande publique ${ }^{4}$.

\footnotetext{
Question écrite $\mathrm{n}^{\circ} 16901, J O R F$, Sénat, 31 Mars 2005 ; réponse du ministère de l'économie, JORF, Sénat, 19 mai 2005.

$4 \quad$ Le cas du contrat de partenariat de Villemandeur, relatif à la construction d'un collège, est révélateur des difficultés juridiques potentielles relatives à la mobilisation du critère d'urgence. Ce dernier a en effet été invalidé par le tribunal administratif, avant d'être réhabilité par la Cour administrative d'appel de
} 


\section{Le motif fondé sur l'efficacité}

La loi de juillet 2008 introduit un troisième motif susceptible de justifier le recours au contrat de partenariat. Ce motif a une dimension économique nettement plus marquée puisqu'il introduit explicitement un critère d'efficacité. Le Conseil constitutionnel a conforté ce point (DC $\mathrm{n}^{\circ}$ 2008-567), en reconnaissant que le recours au contrat de partenariat peut être justifié par des considérations fondées sur le bon usage des deniers publics, même en l'absence d'urgence et/ou de complexité. Cela suppose que cette solution présente un bilan plus favorable que les formes alternatives, en particulier en termes de coût global actualisé et/ou de performance attendue.

Bien que visant des objectifs louables, la mise en œuvre de ce critère reste à ce jour problématique. Justifier le recours à une forme contractuelle au motif de son efficacité supérieure avant qu'elle ne soit effectivement mise en œuvre est en effet une entreprise complexe, susceptible d'ouvrir la voie à de nombreux contentieux. C'est d'autant plus vrai que nombre de projets comprennent des enjeux politiques et sociaux, ce qui est parfaitement légitime, susceptibles d'influencer certains choix méthodologiques. Les enjeux entourant le recours à ce critère d'efficacité se révèlent donc considérables. Les développements qui suivent ont pour objectif principal d'éclairer les facettes de ce concept dans le cadre de l'évaluation préalable.

\section{L'EVALUATION COMPAREE, ABSTRACTION FAITE DES RISQUES}

Nous proposons d'examiner les composantes de ce critère d'efficacité de façon séquentielle. Dans une première étape, nous examinons de possibles fondements à une

Nantes par un arrêt du 23 janvier 2009. Ces aléas, sont à relier à l'analyse des coûts de transaction présentée dans la section 2. Pour de plus amples développements juridiques sur les critères d'urgence et de complexité, voir Leraut (Jonathan), «Réflexions sur les conditions d'urgence et de complexité justifiant le recours au contrat de partenariat », Bulletin juridique des contrats publics, $\mathrm{n}^{\circ} 42,2005, \mathrm{p} .343$. 
comparaison des solutions alternatives en faisant abstraction des risques. Il s'agit alors d'identifier et d'évaluer les caractéristiques structurelles de ces alternatives. Une deuxième étape consiste à introduire explicitement le risque, en cernant les diverses formes qu'il peut prendre, ce qui nous amènera à en proposer une typologie, et en préciser ses conséquences contractuelles.

Nous nous concentrons pour le moment sur la première étape, retenant la question des risques pour la section suivante. Après un bref rappel des enseignements issus de la théorie des coûts de transaction, qui a fait émerger l'idée que l'efficacité d'une mode organisationnel est fonction de son adéquation aux caractéristiques des transactions auxquelles il va servir de support (alignment principle), nous mettons en exergue l'importance et les difficultés d'une reconstitution du coût complet des solutions alternatives, certains des problèmes que soulèvent les hypothèses retenues dans le cadre de ces évaluations, et les difficultés propres à la prise en compte des revenus tiers et des aspects socio-économiques.

\section{Le principe d'alignement}

La théorie économique des organisations, en particulier la branche qui porte sur les accords contractuels, a étudié de façon extensive la question de la comparaison des modes alternatifs d'organisation ${ }^{5}$. Sur ce point, la théorie des coûts de transaction initiée par Coase (1988) et O.E. Williamson ${ }^{6}$ a été particulièrement féconde et a été étoffée sur de nombreux

5 Ménard (Claude), L’Economie des organisations, Paris, Repères, La Découverte, 2004 ; Ménard (Claude), «A New Institutional Approach to Organization », in Ménard (Claude) and Shirley (Mary) eds., Handbook of New Institutional Economics, Boston-New York-Berlin-Dordrecht, Springer, p. 281-318, 2005 ; Saussier (Saussier) et Yvrande-Billon (Anne), Economie des coûts de transaction, Paris, Repères, La Découverte, 2007.

6 Williamson (Oliver E.), The Economic Institutions of Capitalism. Firms, Markets, Relational Contracting, New-York, The Free Press, 1985 ; Williamson (Oliver E.), The Mechanisms of Governance, Oxford, Oxford University Press, 1996. 
aspects par la théorie dite des « contrats incomplets ${ }^{7}$. L'essentiel des travaux inspirés de ces modèles concerne cependant les transactions entre opérateurs privés. Les études consacrées aux choix organisationnels des autorités publiques sont plus récentes et beaucoup moins nombreuses ${ }^{8}$.

Ces travaux convergent sur l'idée que le choix du mode contractuel susceptible de satisfaire le critère d'efficacité n'est pas aléatoire: il existe des arrangements comparativement mieux adaptés que d'autres pour organiser certains types de transaction. Face à la diversité des modes alternatifs, la théorie des coûts de transaction propose en particulier le critère de «l'alignement », c'est-à-dire d'un arrangement par rapport aux caractéristiques des transactions considérées. Si on fait l'hypothèse que les agents cherchent à minimiser leurs coûts, ils sont alors fortement incités à choisir l'arrangement le plus adéquat par rapport à ces caractéristiques, notamment le degré de spécificité des investissements requis ${ }^{9}$ et l'incertitude qui entoure le contrat. C'est ce que O.E. Williamson a proposé d'appeler dès 1985 le principe d'alignement discriminant, défini comme : « the assignment of

7 Hart (Oliver D.) et Moore (John), « Incomplete Contracts and Renegotiation », Econometrica, $\mathrm{n}^{\circ} 56(4), 1988, \mathrm{pp} .755-785$.

$8 \quad$ Hart (Oliver D.), Shleifer (Andrei) et Vishny (Robert W.), « The Proper Scope of Government : Theory and Application to Prisons », The Quarterly Journal of Economics, n²112, 1997, pp. 1127-1161 ; Bajari (Patrick) et Tadelis (Steven), «Incentives versus Transaction Costs : A Theory of Procurement Contracts », Rand Journal of Economics, n`32(3), 2001, pp. 387-407 ; Ménard (Claude) et Saussier (Stéphane), « Contractual Choice and Performance », in Brousseau (Eric) et Glachant (Jean-Michel) eds., The Economics of Contracts. Theory and Applications. Cambridge (UK), Cambridge University Press, p.440-462, 2002.

9 Les investissements spécifiques sont des investissements non redéployables sur d'autres transactions (par exemple d'autres contrats), ou rédéployables mais avec des coûts très importants. Ils peuvent tenir aux caractéristiques physiques, aux propriétés requises du capital humain, etc. (Voir Ménard (Claude), L'Economie des organisations, op . cit., pp. 24-25, et Saussier (Stéphane) et Yvrande-Billon (Anne), Economie des coûts de transaction, op. cit., pp. 17-22 ). 
least-cost governance structures to manage transactions ${ }^{10}$. De très nombreux tests empiriques sont venus étoffer cette proposition ${ }^{11}$.

Si on adopte cette optique, on attendra de l'évaluation préalable qu'elle accorde la plus grande attention aux principales caractéristiques des transactions envisagées, notamment les investissements en actifs spécifiques nécessaires à la réalisation du service considéré ainsi que les facteurs d'incertitude. Alors que cette dernière fait déjà l'objet d'attentions particulières dans les évaluations préalables existantes, mais dans des conditions à discuter (voir notre section 3), la spécificité des investissements requis n'a pas donné lieu à la même attention. Or cela nous paraît un élément clé dans la prise en compte comparative de l'ensemble des coûts.

\section{La reconstitution du coût complet des formes alternatives}

Ces caractéristiques, en particulier la nature des investissements requis, jouent en effet un rôle déterminant dans la comparaison des coûts et donc dans la capacité de mettre en œuvre le principe d'alignement, c'est-à-dire d'éclairer le choix de la solution organisationnelle adéquate. Il convient donc de rappeler succinctement différentes composantes qu'une évaluation préalable devrait prendre en compte, même si elle ne peut le faire que de façon partielle puisqu'il s'agit par définition d'une analyse préparatoire. La combinaison des coûts de transaction et des coûts de financement en constitue le cœur. Leur prise en compte, y compris dans le cas des solutions publiques constituent donc un enjeu important. Nous nous concentrons sur ces points dans les développements qui suivent. En revanche, nous

10 Williamson (Oliver E.), The Economic Institutions of Capitalism. Firms, Markets, Relational Contracting, op. cit., p. 41 ; Williamson (Oliver E.), The Mechanisms of Governance, op. cit., p. 378.

11 Pour un bilan récent dans le cas des arbitrages privés, voir Klein (Peter G.), « The Make-orBuy Decisions. Lessons from Empirical Studies », in Ménard (Claude) et Shirley (Mary) eds., Handbook of New Institutional Economics, Berlin-Dordrecht-New York, Springer, 2005, pp. 435-464. Pour des tests économétriques sur des partenariats public-privé, voir Masten (Scott E.), «Transaction Costs, Mistakes, and Performance: Assessing the Importance of Governance », Managerial and Decision Economics, ${ }^{\circ} 14(2), 1993$, pp. 119-129 ou Ménard (Claude) et Saussier (Stéphane), « Contractual Choice and Performance », op. cit. 
n'examinons guère les coûts de construction, d'exploitation et de maintenance, qui sont certes d'une importance majeure dans l'explication des coûts associés à chacune des formes alternatives, mais qui sont d'avantage du domaine technique et présentent a priori moins de difficultés pour l'évaluation économique. Il n'en reste pas moins qu'ils doivent bien évidemment faire l'objet de développements dûment justifiés dans le cadre des évaluations préalables.

\section{Les coûts de transaction}

Les coûts de transaction correspondent à l'ensemble des coûts résultant des dispositifs choisis pour assurer le transfert de droits d'usage, donc, le cas échéant, tant les transferts de droits de propriété que les transferts de droits de décision ${ }^{12}$. En particulier, ils ont trait à l'ensemble des «coûts de contractualisation», c'est-à-dire les coûts d'élaboration et d'exécution de la solution contractuelle retenue.

Ces coûts ont été identifiés par Ronald Coase, ce qui lui a valu le prix Nobel d'économie, et opérationnalisés par Oliver Williamson. La littérature dérivée de ces auteurs distingue les coûts de transaction ex ante (essentiellement les coûts d'information, liés à la recherche de partenaires potentiels, l'identification du partenaire le mieux placé, la recherche des prix adéquats; et les coûts de négociation et de rédaction du contrat, en particulier les clauses de sauvegarde) ; et les coûts de transaction ex post (c'est-à-dire essentiellement les coûts de mise en œuvre du contrat, en particulier les coûts de suivi et de pilotage, les coûts résultant d'éléments inadaptés ou mal calibrés dans ce contrat, les coûts de renégociation qui en résultent, et enfin les coûts provenant d'une rupture éventuelle de la relation contractuelle).

Le développement de ce cadre théorique et les très nombreuses études empiriques auxquelles il a donné lieu permettent désormais une bonne identification des déterminants de ces coûts et fournissent des éléments en vue de leur prise en compte. Certes, ces évaluations empiriques se heurtent à certaines difficultés méthodologiques, identifiées d'ailleurs assez tôt 
dans la littérature ${ }^{13}$, par exemple la détermination des coûts de solutions alternatives qui restent virtuelles, comme c'est le cas dans l'évaluation préalable. Néanmoins des outils existent désormais qui permettent d'identifier et de mesurer une partie non négligeable de ces coûts.

Ainsi, un certain nombre d'études suggèrent-elles que les coûts de transaction ex ante sont plus élevés pour les partenariats public-privé, en particulier les contrats de partenariat, que pour les autres solutions contractuelles. Une étude récente de F. Marty et A. Voisin ${ }^{14}$ suggère que les coûts de réponse à un appel d'offres sont trois fois plus élevés pour des contrats de type Private Finance Initiative (PFI) que lors de la passation de marchés publics habituels. T. Välila estime de son côté que les seuls coûts de conseils ex ante pour les PFI anglais peuvent atteindre jusqu'à $10 \%$ de la valeur d'un projet et représentent en moyenne 3,7\% pour les projets les plus importants ${ }^{15}$. Dans le cadre français, un rapport au commissariat du plan fait état des craintes quant au niveau réel des coûts de transaction ${ }^{16}$. En associant les coûts d'évaluation préalable, de renégociation, d'indemnisation des candidats, de recherche d'information, de contrôle de l'opérateur, le montant supposé des coûts de transaction défavoriserait le recours au contrat de partenariat pour les opérations de faible ampleur. Toujours selon le rapport Baumstark, le critère déterminant serait celui de l'expertise

13 Masten (Scott E.), Meehan (James W.) et Snyder (Edwards A.), « The Costs of Organization », Journal of Law, Economics et Organization, 7(1), 1991, pp. 1-25.

14 Marty (Frédéric) et Voisin (Arnaud), « Partnership contracts, project finance and information asymmetries : From competition for the contract to competition within the contract ? », Document de travail $O F C E, \mathrm{n}^{\circ} 06,2008$.

15 Välilä (Timo), «How expensive are cost savings. On the economic of public-private partnerships », European Investment Bank Papers, n¹0(1), 2005, pp. 94-119.

16 Baumstark (Luc), Huge (Albane), Marcadier (Catherine), Maubert (Catherine), Commissariat Général du Plan, Partenariats Public-Privé et Actions Locales, Paris, La Documentation française, 2005. Ces études, encore trop peu nombreuses, demandent à être confortées ... ou contestées. En effet elles se heurtent aux difficultés qu'il y a, en l'état actuel des choses, à estimer les coûts de transaction de la solution publique, ce qui tend évidemment à biaiser les résultats sans qu'on sache trop dans quelle direction. Pour une approche plus favorable à la participation du secteur privé de ce point de vue, voir Banque Mondiale, 2008 Annual Report. Helping to eliminate poverty and achieve sustainable development through public-private partnerships in infrastructure, Public-Private Infrastructure Advisory Facility, 2009. 
disponible en interne chez la personne publique. En conséquence, le développement du contrat de partenariat serait conditionné par l'apprentissage des personnes publiques et la réduction du risque juridique lié au dispositif.

Afin de réduire les coûts de transaction, et d'améliorer ainsi la qualité intrinsèque des évaluations préalables, plusieurs pistes peuvent être envisagées. Ainsi, serait-il sans doute avantageux de mener les évaluations préalables en deux étapes. La première aurait pour objectif de justifier la forme contractuelle la plus adaptée à l'aide d'une analyse simplifiée des variables clés identifiées dans cet article. La seconde étape viserait l'évaluation des autorisations d'engagement à prévoir à travers notamment une analyse financière plus fine du coût des facteurs et en premier lieu des risques.

Des efforts accrus visant à conforter la légitimité des évaluations préalables, par exemple en renforçant la transparence dans le choix des experts et l'indépendance des organismes en charge des évaluations, participeraient aussi à réduire les coûts de transaction associés à cette procédure. La diversification des expertises (techniques, économiques, financières, juridiques), lorsque la taille du projet le permet, et la responsabilisation des acteurs eu égard à la réalisation des projets seraient également susceptibles de réduire les coûts de transaction, notamment en diminuant le risque de contentieux. Enfin, toujours dans la même optique, on pourrait conférer à l'évaluation préalable le rôle d'outil pour le pilotage des projets. L'utilisation des conclusions de l'évaluation pour guider la négociation du marché et pour encadrer les conditions d'exécution du contrat pourrait fortement contribuer à réduire les écarts entre ces différentes étapes. Même si une certaine flexibilité doit être conservée afin de s'adapter aux conclusions de la procédure de passation et aux contingences intervenant après la signature du marché, il serait sans doute judicieux d'explorer les avantages d'une rigidification partielle des conditions de signature et d'exécution des contrats afin d'améliorer la crédibilité des évaluations préalables.

\section{Les coûts de financement}

Les conditions du mode de financement et ses coûts prédominent fortement dans les évaluations préalables existantes. Il y a évidemment à cela de bonnes raisons. La modélisation 
de la structuration financière du projet est essentielle dans le calcul de son coût. Il s'agit d'apprécier, à partir des coûts d'investissement, d'éventuels revenus anticipés, et des sources de financement, la faisabilité financière d'un projet, en particulier la capacité à obtenir le soutien bancaire (la « bancabilité »).

Les ratios classiques d'appréciation de la solidité financière d'un projet sont susceptibles d'être mobilisés dans ce cadre, par exemple, le rapport dette sur fonds propres, le ratio de couverture de la dette par les revenus du projet (DSCR : Debt Service Cover Ratio), calculé pour chaque année du projet, ainsi que le ratio actuariel de couverture de la dette, pouvant être affiné en calculant la valeur actuelle nette sur la durée de vie de la dette (LLCR : Loan Life Cover Ratio). Du point de vue de la personne publique, l'utilisation de tels ratios financiers n'a pas pour but de déterminer la structure optimale de financement, mais d'évaluer la faisabilité d'un montage en partenariat public-privé.

Le choix des sources de financement dépend d'une analyse multicritère reposant à la fois sur la taille du projet, sa complexité, les garanties requises, ou encore les conditions du marché financier. Les deux montages généralement envisagés pour un partenariat public-privé sont le financement de projet et le crédit-bail. Le financement de projet est réservé à des projets d'un montant important, le seuil communément admis pour qu'un tel montage soit envisageable étant 20 millions d'euros. Pour les projets de taille plus modeste, le financement en crédit-bail ou par une formule de financement corporate est plus adapté et moins coûteux. Quel que soit le montage modélisé a priori, la comparaison avec un financement public classique implique la prise en compte des différentes sources de financement disponibles, à savoir les subventions éventuelles par d'autres personnes publiques, en particulier $a b$ initio, permettant de réduire significativement les montants empruntés et le coût global du financement.

Dans le cas d'un montage avec une société dédiée (dite «société de projet»), on sera particulièrement attentif au ratio capital / fonds propres, dont le passif est essentiellement formé de dettes bancaires, et marginalement de capitaux propres apportés par les membres du groupement signataire dans des proportions variables au cours du contrat et déterminées en 
fonction du niveau de risque, de la taille et de la nature du projet ; à la dette subordonnée, accordée par les actionnaires ou investisseurs tiers et considérée comme des quasi-fonds propres ; ainsi qu'à la dette bancaire senior, la durée des prêts aménageant généralement une période résiduelle après le remboursement prévu du crédit en cas de rééchelonnement de la dette.

Dans le cas d'un financement bancaire, on distingue la phase de développement et de construction, pendant laquelle sont effectués les tirages, et la phase d'exploitation pendant laquelle s'effectue le remboursement; la cession de créance, par mécanisme de cession «Dailly » ${ }^{17}$ notamment, pour diminuer les marges d'intérêt sur une portion significative de la dette senior ; l'émission obligataire, adaptée pour des projets de taille très importante et de durée longue ; le financement par crédit-bail, adapté aux projets de taille petite ou moyenne ${ }^{18}$.

L'ensemble de ces modalités, et des modélisations qui les accompagnent, reste évidemment très sensible aux hypothèses émises, particulièrement au stade encore préliminaire de l'évaluation préalable, ce qui fragilise beaucoup celle-ci, point sur lequel nous reviendrons dans la sous-section suivante.

\section{La reconstitution du coût des solutions publiques}

La reconstitution du coût global anticipé du recours à un opérateur privé devrait être relativement aisée dans le cadre des contrats de partenariat dans la mesure où ce coût correspond à la somme des loyers prévus et versés tout au long de la vie du marché par la personne publique, auquel il convient d'ajouter les coûts de transaction associés à la passation et au suivi du marché considéré. Des marges correspondant aux conséquences d'éventuelles renégociations intervenant ex post peuvent aussi être introduites dans le calcul.

$17 \quad$ La cession Dailly est un accord passé entre les banques et la personne publique selon lequel cette dernière s'engage à verser une part du loyer directement aux banques ayant participé au financement du projet. Cet arrangement financier réduit le coût du financement grâce à un taux d'emprunt plus intéressant. 
La difficulté est nettement accrue lorsqu'il s'agit de reconstituer le coût complet de la prise en charge directe par une entité publique (par exemple une régie municipale). Le rapport de la Cour des comptes de mai 2008, sur les résultats et la gestion budgétaire le l'État pour l'exercice 2007, souligne que « les gestionnaires ont encore une vision partielle des coûts des actions qu'ils mettent en œuvre. Leur analyse est en effet réduite à la collecte de chiffres, en général très partiels, et bien souvent ne constitue pas un outil d'aide à la décision ». L'évaluation préalable devrait contribuer dans cette perspective à limiter une asymétrie d'information importante tenant à la méconnaissance des coûts publics. Celle-ci résulte de l'existence de coûts cachés et d'incertitudes que les méthodes de gestion et de comptabilité des collectivités publiques ne prennent pas en compte. Or c'est un aspect essentiel de l'exercice imposé par l'évaluation préalable, dont on attend :

- Une évaluation des coûts indivis du secteur public qui doivent être impartis au projet. En particulier, comment comptabiliser les frais généraux liés à l'élaboration, la réalisation et le suivi du projet? Quels sont les coûts de gestion des ressources humaines qui doivent lui être imputés ?

- La prise en compte du coût des pensions des personnels publics et de l'amortissement des biens.

- Une appréciation aussi exacte que possible du coût du financement public. Par convention, ce coût correspond, lorsque l'État est le mandant, au taux des obligations assimilables du trésor (OAT) de même durée que le projet considéré ; et, lorsque ce sont les collectivités territoriales qui sont le mandant, au coût moyen de l'endettement

18 Pour de plus amples développements sur le financement des projets, voir Christophe (Jean), Marty (Frédéric) et Voisin (Arnaud), «Le financement des partenariats public-privé », Contrats publics actualité de la commande et des contrats publics, $n^{\circ} 65,2007$, pp. 28-35. 
de la collectivité considérée, qui varie en fonction notamment de sa notation financière ${ }^{19}$.

Outre la prise en compte explicite, même de façon partielle, des coûts de transaction et des coûts de financement, en particulier en ce qui concerne les solutions publiques, il y aurait lieu de systématiser les évaluations ex post des évaluations préalables afin d'en apprécier la pertinence et d'en expliquer les éventuelles limites. La constitution d'une base de données systématique sur les évaluations préalables et sa mise à disposition publique pourrait fournir un outil précieux en ce sens et contribuer à terme à une bien meilleure appréciation des coûts des solutions alternatives, en particulier des coûts de transaction.

\section{Les hypothèses retenues}

Une limite invoquée de façon récurrente par les experts concerne la faiblesse des hypothèses sur lesquelles repose l'analyse comparative, que la non transparence des grilles d'analyse utilisées accentue, de sorte qu'elles peuvent facilement être remises en cause par des contre-expertises. Il en résulte une peur du contentieux qui constitue un frein au recours aux contrats de partenariat. La formulation explicite dans le document d'évaluation d'hypothèses claires et bien motivées représenterait ainsi un progrès considérable. Le manque actuel de lisibilité des hypothèses utilisées concerne particulièrement :

(i) L'estimation des délais de construction, et des écarts entre délais en contrat de partenariat et en montage «classique », du type maîtrise d'ouvrage public ou délégation de service public. La plupart des évaluations préalables se fondent sur un avantage supposé du

19 Ainsi l'écart de taux entre le coût de l'obligation assimilable du trésor et le coût moyen de l'endettement de la collectivité considéré doit-il être pris très au sérieux car il sert à calculer le taux d'actualisation, déterminant lui-même la potentielle rentabilité des projets. Choisir un taux d'actualisation erroné conduirait à sélectionner des projets inopportuns ou sous des formes contractuelles inappropriées. Par exemple, un taux d'actualisation trop élevé inciterait à favoriser les contrats de partenariat dans la mesure où le paiement des investissements y est lissé dans le temps. 
contrat de partenariat dans ce domaine, mais demeurent vagues pour le justifier, se référant occasionnellement aux « expériences étrangères ».

(ii) Les hypothèses retenues en matière de montage financier. Les hypothèses sur les taux de marge attendus sur la dette projet, sur la dette subordonnée, ou sur les commissions bancaires peuvent facilement être remises en cause, en particulier pour les projets de moindre ampleur. Elles semblent en effet calquées sur des projets similaires plus anciens, sans vraiment tenir compte des évolutions des marchés financiers et des conditions générales de financement. On notera aussi la faiblesse des justifications sous-tendant les lois de probabilité associées aux différents risques, et à leurs interactions, susceptibles de se matérialiser durant la vie des projets. La constitution d'une base de données devrait là encore contribuer à une plus grande fiabilité.

(iii) Les économies attendues via un montage en contrat de partenariat en matière de coûts d'exploitation ou de maintenance. Là encore, les justifications apparaissent très sommaires, et les évaluations ne font que rarement apparaître les effets d'hypothèses moins favorables au contrat de partenariat, par exemple en fonction des critères de mesure retenus (comme la valeur actuelle nette ou Value At Risk ${ }^{20}$ ).

\section{Les revenus tiers}

Une caractéristique essentielle des contrats de partenariat concerne la possibilité de lever des revenus tiers grâce à la location, à des clients autres que celui ayant signé le marché, de l'équipement et/ou des services associés au contrat. Cette opportunité provient directement de l'approche capacitaire favorisant davantage l'existence de tels revenus que l'approche patrimoniale, par exemple du type marché public. Même si les revenus tiers restent d'une

$20 \quad$ La Value at Risk fait référence au montant des pertes pouvant être subies à travers l'exécution du projet. Cette valeur est notamment déterminée en corrélation étroite avec le niveau de confiance et l'horizon temporel retenus. 
importance modérée, voire tout à fait marginale à l'heure actuelle, il convient de rappeler qu'ils sont susceptibles d'alléger significativement le coût pour l'opérateur privé et pour la personne publique concernée. Aussi peut-on s'attendre à une prise en compte rigoureuse de ces revenus dans l'évaluation préalable.

Les premiers retours d'expérience sur les contrats signés invitent toutefois à la prudence quant à l'estimation des revenus tiers. En effet leur montant effectif s'avère souvent décevant. Sans nécessairement remettre en cause la créativité des partenaires privés dans le développement d'activités annexes, il convient de tenir compte des difficultés, en particulier juridiques, liées aux usages annexes. On attend donc de l'évaluation préalable:

- L'identification d'éventuels revenus tiers : l'équipement faisant l'objet du contrat sera t-il utilisé à $100 \%$ par le commanditaire ? L'équipement peut-il être cédé à des organisations tierces? Des organisations tierces sont-elles susceptibles d'être intéressées par ledit équipement ou service?

- L'évaluation des revenus tiers éventuels pour toute la durée de vie du marché : quel est le coût journalier ou mensuel associé à l'utilisation de cet équipement? Quelle est la disposition à payer des organisations tierces potentiellement intéressées par l'équipement considéré ?

- L'appréciation de la part des revenus tiers susceptible de contribuer à la réduction du coût global pour la personne publique : quelle est la distribution prévue des gains associés à ces éventuels revenus ? Participent-ils à réduire le coût global d'acquisition pour la partie publique, ou constituent-ils une entrée nette pour le seul contractant privé ?

- La prise en compte des conditions à remplir pour garantir au moins jusqu'à un certain point les revenus tiers, lorsque le cas s'applique, et la répartition des risques (et des recettes) qui devraient en découler entre autorités publiques et partenaires privés. 


\section{Les aspects socio-économiques}

Un reproche fréquemment adressé aux évaluations préalables est d'omettre les aspects socio-économiques. Elles s'en tiendraient au seul calcul financier alors que le critère d'efficacité exigerait une vision plus large, prenant en compte les utilités sociales attendues. Cette lacune est d'autant plus dommageable que pour nombre d'acteurs, l'avantage comparatif réel du contrat de partenariat s'évalue d'abord en termes socio-économiques : il permettrait la réalisation de projets répondant à des besoins et qui n'auraient pu voir le jour, ou qui auraient dû être retardés sans cette forme contractuelle innovante. Les contrats de partenariat seraient de plus susceptibles, sous certaines conditions, d'offrir une qualité de service supérieure à celle des modes d'organisation alternatifs. Aussi, bien que la prise en compte des aspects socio-économiques dans l'évaluation préalable ne soit pas obligatoire, dans la mesure où ils sont supposés introduits en amont dans la décision de réaliser le projet considéré, s'avère-t-il utile d'en rendre compte dans la comparaison des solutions alternatives envisagées pour répondre au besoin exprimé par la personne publique. Cela participerait en effet à rendre l'évaluation plus complète, et plus convaincante.

\section{LA PRISE EN COMPTE DES RISQUES DANS L'EVALUATION PREALABLE}

Dans ce qui précède, nous avons fait largement abstraction du facteur « risques ». Or ceux-ci constituent évidemment une dimension essentielle de toute évaluation préalable tant leur impact peut être significatif, et varier selon les solutions retenues. Nous nous concentrons ici sur les types de risques à envisager et leur répartition, laissant la question de leur évaluation à une analyse ultérieure. 


\section{La classification des risques}

Dans tout projet, les événements susceptibles de creuser l'écart entre les attentes et les réalisations sont nombreux et de nature diverse. Nous voudrions ici mettre l'accent sur l'importance que peut avoir, pour l'évaluation préalable comme pour le contrat lui-même, le recours à une classification homogène et solidement établie des types de risques à prendre en compte. Une telle typologie peut remplir deux fonctions principales. D'abord, elle contribuerait à structurer l'évaluation préalable en fournissant un instrument de collecte et d'organisation de l'information sur les risques potentiels ${ }^{21}$. Il devrait en résulter plus de transparence. Ensuite, une typologie des risques adéquate devrait faciliter la rédaction et l'exécution des contrats, en particulier concernant l'allocation des risques.

Pour satisfaire ces exigences, une telle typologie doit reposer sur un cadre de référence homogène, proposant une partition où un même événement ne puisse être classé dans plusieurs catégories. Elle doit aussi pouvoir s'appliquer tant à la phase précontractuelle (analyse préliminaire et évaluation préalable) qu'à la phase contractuelle (passation, rédaction et exécution du marché). Ceci devrait en effet permettre d'éviter les redondances et la multiplication des entrées «risques » aux différentes étapes du projet, et devrait favoriser la cohérence entre le projet et son suivi, rapprochant les conditions d'exécution des marchés des anticipations énoncées dans l'évaluation préalable. On faciliterait ainsi le rôle de l'évaluation préalable comme outil de pilotage des projets, les mêmes notions servant aux différentes étapes de la vie de ceux-ci. Enfin, la typologie doit éviter les ambiguïtés, de manière à faciliter une véritable comparaison des solutions alternatives, tout en tenant compte des contraintes d'information qu'impose l'effort d'évaluation des risques. Il convient donc de retenir un nombre limité de catégories de risques, capable en même temps de s'ajuster en fonction du degré de complexité de la transaction envisagée.

21 Chrissis (Mary Beth), Konrad (Mike) et Shrum (Sandy), CMMI. Guidelines for Process Integration and Product Improvement, Addison-Wesley, 2004, p. 500. 
Trois solutions peuvent être envisagées pour fonder une telle typologie. Une première possibilité, qui prévaut dans les évaluations préalables existantes, consiste à classer les risques en fonction de la période pendant laquelle ceux-ci sont susceptibles de se matérialiser à savoir avant, pendant (passation, rédaction) ou après la signature du marché. Cette approche présente l'avantage de faciliter la gestion de projets. Elle se heurte cependant aux problèmes de redondances dans la mesure où un même événement fait l'objet d'évaluations successives selon les moments où il intervient. De telles redondances apparaissent, par exemple, lorsque des risques se matérialisent, en phase de développement mais aussi pendant la production de l'équipement ou de l'infrastructure considéré. L'approche fondée sur le moment d'occurrence des risques consiste dans ces conditions à évaluer ces possibilités à chaque phase. Il en résulte un accroissement des efforts de suivi et de leurs coûts associés.

Une deuxième possibilité consiste à centrer l'analyse sur l'impact supputé des risques. Ceux-ci pouvant conduire à des surcoûts, des surprix, des retards et/ou une réduction de la qualité de l'équipement ou du service. L'analyse privilégie alors les risques financiers, calendaires et techniques. L'avantage de cette approche est de mettre en avant les conséquences des risques sur la rentabilité du projet. Son inconvénient est cependant de faire passer au second plan l'analyse des sources des risques. Or ces dernières sont d'une importance centrale, comme indiqué ci-dessous.

Les développements de l'analyse des risques dans une perspective organisationnelle permettent de dépasser les seuls risques financiers et de fonder une typologie en fonction de l'origine des événements adverses ${ }^{22}$. Cette approche devrait notamment faciliter la répartition des risques entre partenaires dans la mesure où elle conduit assez naturellement à l'identification des responsabilités eu égard aux événements survenant pendant la durée de vie des projets. Conçue pour être transversale aux diverses étapes, une telle typologie devrait

22 Oudot (Jean-Michel), Choix contractuels et performances. Le cas des contrats d'approvisionnement de défense, Thèse de doctorat en sciences économiques, Université Paris I PanthéonSorbonne, 2007, chapitres 2 et 3, http://tel.archives-ouvertes.fr/docs/00/18/71/50/PDF/Oudot_jeanmichel_these.pdf 
permettre de faciliter l'identification et l'allocation des risques, réduisant ainsi les coûts dans la conception, la rédaction et l'exécution des contrats, tout en favorisant la cohérence d'ensemble des projets. Typologie et répartition des risques deviennent ainsi étroitement liés.

Cette approche fondée sur l'origine des risques permet d'en distinguer cinq sources:

Le risque technologique, c'est-à-dire les évènements techniques ou technologiques susceptibles d'affecter la performance de la relation contractuelle. Qu'on pense par exemple à l'émergence d'une innovation plus performante que le produit ou procédé envisagé dans le contrat, au manque de maturité de la technologie utilisée, ou encore à des problèmes de qualité des matériaux.

Le risque organisationnel, c'est-à-dire l'ensemble des événements liés à une défaillance involontaire du titulaire du contrat et/ou de ses partenaires. Le manque de coordination entre le titulaire et les sous-traitants ou encore la défaillance industrielle d'un sous-traitant en fournissent des exemples.

Le risque financier direct, c'est à dire les évènements qui menacent directement l'équilibre financier prévu initialement dans le contrat. C'est le cas lorsqu'il y a modification du coût des matières premières (risque-marché), ou encore non mise à disposition des crédits prévus au moment convenu.

Le risque contractuel, c'est-à-dire les évènements liés à la négociation, à l'écriture et au suivi du contrat. Des exemples en sont le non respect des engagements de l'autorité publique en matière de fourniture de consommations intermédiaires sur laquelle elle avait pris des engagements, ou encore l'opportunisme du titulaire du marché ou de la personne publique.

Le risque institutionnel, enfin, c'est-à-dire les événements résultants de changements des règles du jeu susceptibles d'induire des coûts effectifs différents de ceux anticipés, par exemple l'apparition d'une nouvelle réglementation environnementale ou sociale, ou l'interdiction d'importation d'une consommation intermédiaire prévue contractuellement. 
Bien entendu, tous ces risques n'interviennent pas à toutes les étapes et dans tous les projets. Mais la possibilité de les prendre en compte à toutes les étapes, grâce à la stabilité de la grille d'analyse fournie aux différentes étapes, et ce dès l'évaluation préalable, devrait assurer une grande cohérence dans les procédures d'évaluation, tant ex-ante qu'au cours de la mise en œuvre de la solution retenue. Par ailleurs, il conviendrait d'affiner les méthodologies existantes de manière à prendre en compte et mesurer de façon adéquate ces divers types de risques.

\section{L'allocation des risques}

Une des caractéristiques essentielles des contrats de partenariat est l'attention portée au partage des risques, et donc à la répartition entre partenaires des surcoûts, voire des gains inattendus, résultant de l'exécution du contrat.

Bien que ce soit fondamental, les transferts de risque selon les solutions organisationnelles envisagées font rarement l'objet d'une explication argumentée dans les rapports d'évaluation ${ }^{23}$. La pratique usuelle consiste à comparer l'allocation des risques issue d'un montage en maîtrise d'ouvrage publique à celle résultant d'un contrat de partenariat. La personne publique est alors le plus souvent supposée capable d'imposer le transfert de risques au futur partenaire. Dans cette perspective, le soin apporté à la réflexion sur l'étendue des transferts et leurs conditions d'application est nettement plus marqué dans les projets d'État que dans les projets des collectivités territoriales. Dans les évaluations préalables ayant trait à des projets de taille modeste, le transfert de risques semble correspondre davantage à un exposé de ce qu'il est théoriquement possible de faire supporter au partenaire privé qu'à une adaptation précise au projet évalué. Il en résulte un biais en faveur d'un transfert poussé des risques au partenaire privé. Mais on trouve très rarement un effort d'évaluation et d'allocation

23

L'arrêté du 2 mars 2009 relatif à la méthodologie applicable à l'évaluation préalable et à la mise en œuvre d'une procédure de passation d'un contrat de partenariat rappelle cependant l'importance d'introduire les aspects allocation des risques dans l'évaluation préalable. 
effectives de ces transferts. Certaines études ont également noté une tendance à allouer les risques de sorte que le montant de l'investissement du contrat de partenariat ne figure pas dans la dette publique (contrats de partenariat dits «déconsolidants $)^{24}$. La répartition des risques répondrait alors davantage à des considérations opportunistes, exploitant les modalités comptables de l'investissement de manière à voiler le poids de la dette, qu'à des considérations d'efficacité économique.

La littérature économique sur les contrats, tant la théorie de l'agence que la théorie des coûts de transaction, peut ici apporter un éclairage utile, car elle met l'accent sur l'allocation des risques. On notera entre autres les leçons suivantes, particulièrement pertinentes pour notre sujet car susceptibles de diminuer les coûts de transaction tout en améliorant la transparence des engagements que prennent les parties ${ }^{25}$.

Les transferts de risques devraient être examinés par l'évaluation préalable dans une optique conforme au «principe de responsabilité », chaque partie respectant ses engagements et assumant les conséquences des événements adverses qu'elle peut contrôler ${ }^{26}$.

Lorsque les risques sont d'origine purement exogène, dus par exemple à un changement non anticipé de la réglementation imposant des coûts additionnels, la théorie suggère que ce coût soit supporté par la partie publique. Celle-ci bénéficie en effet d'un coût de support de ce type de risques généralement inférieur à toute organisation privée, en raison de coûts d'opportunité distincts, de canaux de financement différents, et de ses capacités de mutualisation des risques.

$24 \quad$ Marty (Frédéric), «Partenariats public-privé, règles de discipline budgétaire, comptabilité patrimoniale et stratégies de hors bilan », Document de travail OFCE, n²9, 2007.

25 Oudot (Jean-Michel), Choix contractuels et performances. Le cas des contrats d'approvisionnement de défense, op. cit., chapitres 7 et 8 ; Oudot (Jean-Michel), "Choix du type de contrat et performance : le cas des marchés publics de défense », Economie Publique, n²1(2), 2008, pp. 157-182.

26 Holmström (Bengt) et Milgrom (Paul), «Multitask Principal-Agent Analyses : Incentive Contracts, Asset Ownership and Job Design », Journal of Law, Economics et Organization, n' ${ }^{\circ}$, special issue, 1991, pp. 24-52. 
Enfin, une partie des risques susceptibles de se matérialiser sont difficiles à anticiper, et surtout à évaluer, au moment de l'évaluation préalable tout comme à la période de rédaction, voire d'exécution, du contrat. Dans ces cas, la répartition des risques est tout sauf évidente. Dans la typologie proposée plus haut, c'est particulièrement le cas des risques organisationnels ou contractuels. Tout au plus l'évaluation préalable peut-elle alors envisager des règles supplétives, stipulant les obligations des parties en l'absence d'accord explicitant le contraire $^{27}$, soit sur la base du principe de responsabilité lorsqu'il n'y a pas ambiguïté, soit sur la base d'un principe d'équilibre des aléas impliquant un partage des coûts à 50-50 lorsque la complexité du projet rend ambiguë l'origine du risque, principe qu'une littérature récente a mis en exergue ${ }^{28}$.

L'introduction de telles règles, applicables non seulement aux contrats de partenariat mais aussi aux solutions alternatives telles que la maîtrise d'ouvrage public ou la délégation de service public, et des éléments de valorisation qu'elles impliquent au moment de l'évaluation préalable, devrait faciliter le dialogue compétitif lors de la formulation du contrat et faciliter par la suite le règlement des différends, réduisant d'autant les coûts de transaction ex post. Leur prise en compte dès l'évaluation préalable est de plus susceptible de rapprocher les termes de cette évaluation des conditions de signature et d'exécution du contrat, en accroissant d'autant sa crédibilité et sa légitimité.

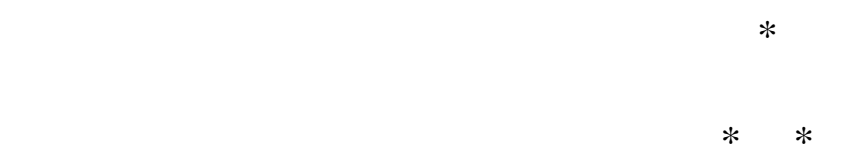

$27 \quad$ Ayres (Ian) et Gertner (Robert), «Filling Gaps in Incomplete Contracts : An Economic Theory of Default Rules », Yale Law Journal, n99, 1989, pp. 30-41 ; Craswell (Richard), "Contract Law : General Theories », in Bouckaert (B.) et De Geest (G.) eds., Encyclopedia of Law and Economics, ${ }^{\circ} 4000$, Edward Elgar, 2001, http://users.ugent.be/ gdegeest/4000book.pdf

28 Oudot (Jean-Michel) et Ménard (Claude), «Opportunisme ou équité ? Le cas des contrats d'approvisionnement de défense », document de travail, 2009. 


\section{Conclusion}

L'évaluation préalable doit être un élément clé de l'arbitrage conduisant la personne publique à choisir ou à rejeter la solution du contrat de partenariat. Sa qualité, et donc sa crédibilité, sont de ce point de vue essentiels si on entend en faire un instrument efficace de la décision publique.

Dans cet article, nous avons examiné le rôle que peuvent jouer à cet effet les préconisations déductibles du principe d'alignement des choix contractuels aux caractéristiques des transactions. Ce principe, développé par la théorie des coûts de transaction, met notamment en avant l'importance de prendre en compte l'incertitude, mais aussi la spécificité des investissements nécessaires à la réponse du besoin exprimé par la personne publique, dans la décision concernant le mode d'organisation. Ces dimensions restent encore prises en compte que de façon partielle dans les évaluations préalables.

Des progrès dans cette direction permettraient de rapprocher les conditions de signature et d'exécution des marchés des anticipations de l'évaluation préalable, évitant les mauvaises surprises et réduisant d'autant les risques de contentieux et, plus généralement, les coûts de transaction ex-post. Plusieurs suggestions ont été formulées en vue de réduire ces coûts de transaction. Parmi celles-ci, la possibilité de mener les évaluations en deux étapes, l'une centrée sur la forme contractuelle la plus adaptée pour répondre à la demande publique, l'autre visant à appréhender au mieux le coût global du marché considéré, a été soulignée. L'introduction systématique de lignes directrices concernant l'allocation des risques dès cette phase précontractuelle constituerait également un moyen d'améliorer qualité et crédibilité des évaluations préalables.

Enfin, il serait sans doute avantageux de donner plus de poids aux conclusions de l'évaluation préalable dans la négociation et l'exécution des marchés. Cela introduirait sans doute une certaine «rigidification » des conditions d'exécution du marché conduisant à une responsabilisation accrue des partenaires, ce qu'il conviendrait cependant de tempérer pour pouvoir tenir compte des nécessaires adaptations, dans la lignée de l'arbitrage stabilité/flexibilité mis en avant par la théorie des coûts de transaction. Toujours dans la 
perspective de cette mise en cohérence accrue des conditions d'exécution du marché avec les conclusions de l'évaluation préalable, il conviendrait d'avoir recours aux mêmes catégories de risques tout au long de la vie des projets, à l'aide d'une typologie fondée sur l'origine des événements, ce qui devrait permettre d'alléger les coûts de transaction tout en facilitant l'allocation des risques.

L'ensemble de ces préconisations vise à préciser les conditions d'utilisation du critère d'efficacité dans l'évaluation préalable dans les contrats de partenariat. Des approfondissements restent à mener, que devrait aider la constitution d'une base de données rassemblant non seulement les conclusions des évaluations préalables mais aussi les conditions d'exécution des marchés, de manière à pouvoir évaluer et améliorer la qualité des études menées lors de la phase précontractuelle et ayant conduit à choisir tel mode d'organisation plutôt que tel autre. La crédibilité des choix publics s'en trouverait renforcée d'autant.

\section{Références}

Ayres (Ian) et Gertner (Robert), "Filling Gaps in Incomplete Contracts: An Economic Theory of Default Rules”, Yale Law Journal, n99, 1989, p.30-41.

Bajari (Patrick) et Tadelis (Steven), "Incentives versus Transaction Costs: A Theory of Procurement Contracts”, Rand Journal of Economics, n³2(3), 2001, p.387-407.

Banque Mondiale, 2008 Annual Report. Helping to eliminate poverty and achieve sustainable development through public-private partnerships in infrastructure, Public-Private Infrastructure Advisory Facility, 2009.

Baumstark (Luc), Huge (Albane), Marcadier (Catherine), Maubert (Catherine), Commissariat Général du Plan, Partenariats Public - Privé et Actions Locales, Paris, La Documentation française, 2005. 
Christophe (Jean), Marty (Frédéric) et Voisin (Arnaud), « Le financement des partenariats public-privé », Contrats publics - actualité de la commande et des contrats publics, $\mathrm{n}^{\circ} 65$, 2007, p.28-35.

Chrissis (Mary Beth), Konrad (Mike) et Shrum (Sandy), CMMI. Guidelines for Process Integration and Product Improvement, Addison-Wesley, 2004.

Coase (Ronald H.), "The Nature of the Firm: Origin, Meaning, and Influence", Journal of Law, Economics \& Organization, n4(1), 1988, p.3-59.

Cour des comptes; Résultats et gestion budgétaire de l'État - exercice 2007, Paris, La Documentation française, 2008.

Craswell (Richard), "Contract Law: General Theories", in Bouckaert B. et De Geest G. (Eds.), Encyclopedia of Law and Economics, n4000, Edward Elgar, 2001, http://users.ugent.be/ gdegeest/4000book.pdf.

Hart (Oliver D.) et Moore (John), "Incomplete Contracts and Renegotiation", Econometrica, $\mathrm{n}^{\circ} 56(4), 1988, \mathrm{p} .755-785$.

Hart (Oliver D.), Shleifer (Andrei) et Vishny (Robert W.), "The Proper Scope of Government: Theory and Application to Prisons", The Quarterly Journal of Economics, $\mathrm{n}^{\circ} 112,1997, \mathrm{p} .1127-1161$.

Holmström (Bengt) et Milgrom (Paul), "Multitask Principal-Agent Analyses: Incentive Contracts, Asset Ownership and Job Design", Journal of Law, Economics et Organization, $\mathrm{n}^{\circ} 7$, special issue, 1991, p.24-52.

Klein (Peter G.), “The Make-or-Buy Decisions. Lessons from Empirical Studies”, Dans Ménard (Claude) et Shirley (Mary) (eds.), Handbook of New Institutional Economics, BerlinDordrecht-New York: Springer, 2005, p.435-464.

Leraut (Jonathan), «Réflexions sur les conditions d'urgence et de complexité justifiant le recours au contrat de partenariat », Bulletin juridique des contrats publics, $\mathrm{n}^{\circ} 42,2005$, p.343 
Marty (Frédéric), « Partenariats public-privé, règles de discipline budgétaire, comptabilité patrimoniale et stratégies de hors bilan », Document de travail OFCE, n²9, 2007.

Marty (Frédéric) et Voisin (Arnaud), "Partnership contracts, project finance and information asymmetries: From competition for the contract to competition within the contract?", Document de travail OFCE, n06, 2008.

Masten (Scott E.), "Transaction Costs, Mistakes, and Performance: Assessing the Importance of Governance", Managerial and Decision Economics, n¹4(2), 1993, p.119-129.

Masten (Scott E.), Meehan (James W.) et Snyder (Edwards A.), "The Costs of Organization”, Journal of Law, Economics et Organization, 7(1), 1991, p.1-25.

Ménard (Claude), L’Economie des organisations, Paris, Repères, La Découverte, 2004.

Ménard (Claude), “A New Institutional Approach to Organization”, in Ménard (Claude) and Shirley (Mary) (eds.), Handbook of New Institutional Economics. Boston-New YorkBerlin-Dordrecht: Springer, p.281-318, 2005.

Ménard (Claude) et Saussier (Stéphane), "Contractual Choice and Performance”, dans Brousseau (Eric) et Glachant (Jean-Michel) (eds.), The Economics of Contracts. Theory and Applications. Cambridge (UK): Cambridge University Press, p.440-462, 2002.

Oudot (Jean-Michel), Choix contractuels et performances. Le cas des contrats d'approvisionnement de défense, Thèse de doctorat en sciences économiques, Université Paris I Panthéon-Sorbonne, 2007.

http://tel.archives-ouvertes.fr/docs/00/18/71/50/PDF/Oudot_jean-michel_these.pdf

Oudot (Jean-Michel), «Choix du type de contrat et performance : le cas des marchés publics de défense », Economie Publique, n²1(2), 2008, p.157-182.

Oudot (Jean-Michel) et Ménard (Claude), «Opportunisme ou équité ? Le cas des contrats d'approvisionnement de défense », document de travail, 2009. 
Saussier (Stéphane) et Yvrande-Billon (Anne), Economie des coûts de transaction, Paris, Repères, La Découverte, 2007.

Välilä (Timo), "How expensive are cost savings. On the economic of public-private partnerships”, European Investment Bank Papers, n¹0(1), 2005, p.94-119.

Williamson (Oliver E.), The Economic Institutions of Capitalism. Firms, Markets, Relational Contracting, New-York, The Free Press, 1985.

Williamson (Oliver E.), The Mechanisms of Governance, Oxford, Oxford University Press, 1996.

Les auteurs:

Claude Ménard est professeur d'économie à l'Université de Paris Panthéon-Sorbonne (Centre d'Economie de la Sorbonne, 106-112 boulevard de l'Hôpital, 75647 Paris cedex 13, menard@univ-paris1.fr).

Jean-Michel Oudot est chargé d'études économiques à la Direction des affaires financières du ministère de la défense, chercheur associé à l'Université de Paris Panthéon-Sorbonne et enseignant à Sciences-Po Paris (Ministère de la défense, DAF/QEFI, 14 rue Saint-Dominique 75007 Paris ; oudot@univ-paris1.fr). 